\title{
Microprocessors in the laboratory: Dedicated computation and time-shared peripherals with REMOTE-11
}

\author{
MARSHALL M. HAITH \\ University of Denver, Denver, Colorado 80208 \\ and \\ BRUCE B. PLATT \\ Digital Equipment Corporation, Englewood, Colorado 80110
}

\begin{abstract}
This article describes a new solution to the dilemma faced by investigators who need dedicated computers but cannot afford the cost of expensive peripherals. An available softwarehardware combination is described that permits users to timeshare expensive peripherals but to have a dedicated microprocessor at their disposal.
\end{abstract}

The advent of the microprocessor appears to have eliminated the last major hurdle to wholesale replacement of the time-honored relays, timers, counters, and recording equipment that have characterized the experimental psychology laboratory for the last several decades. Although the potential of high-speed computers for performing laboratory functions has been recognized for some time, equipment cost has been a major problem. Microprocessors provide a solution to this problem, as they possess control power well in excess of the usual laboratory demands and, at the same time, are quite inexpensive.

The term "microprocessor" covers a broad array of products available on today's market. Basically, microprocessors are general-purpose computers constructed on very small "chips"; frequently, the computational and control modules and some memory can be housed on a single printed circuit card. Although the potential of these modules is fascinating, the laboratory scientist who uses computers for stimulus presentation, data collection, and analysis needs to concern himself with computer systems, not chips. This concern is relevant, because other parts of the system can be expensive.

In this paper we describe an approach that permits the laboratory scientist to capitalize on recent price reductions of computers and at the same time to deal with the problem of expensive computer peripherals. Several computer users can enjoy the advantage of inexpensive, stand-alone high-powered microprocessors

The authors thank Mrs. Priscilla Ray for the gift of the two microprocessors described here and acknowledge the University of Denver Biomedical Research Support Fund, which made possible the purchase of the PDP-11/10 computer. Research conducted with the facility and technician time required for interfacing were supported by NIMH Grant MH23412 and NICHHD Grant HD07232 to Marshall Haith and NICHHD postdoctoral fellowship HD05247 to Bruce Platt. Finally, we thank Betty Richardson for preparing the manuscript. while sharing the cost of expensive peripherals. A readily available, currently operative system is described.

\section{SYSTEM DESCRIPTION}

A computer system requires many elements in addition to the microprocessor itself, which serves as the "brain" of the system. Thus, the fact that the "brain" on a single chip may now cost $\$ 17.50$, whereas only 5 years ago equivalent "brains" cost $\$ 10,000$, may help. But it is equally relevant that the input, output, and storage devices required for a complete system are still fairly expensive, perhaps beyond the range of the single laboratory user. Additionally, programs must be written to command the small laboratory computer system. Larger computer systems with powerful operating systems and language processors may be required to make such program preparation feasible.

One possible solution to these problems has been used at the University of Denver. We have one mediumsized system, well equipped with costly storage and input-output (I/O) devices and extensive software. Individual laboratory users can use this facility to prepare computer programs with the aid of its operating system and language processors. These programs can then be run on individual inexpensive laboratory microprocessors that are satellites to the larger host system. All of the expensive peripherals on the host (e.g., disks, line printer) are available to each of the microprocessors on a demand basis. Thus, users have the advantage of dedicated computers to run their laboratory experiments, while avoiding the duplication of necessary but expensive peripherals. Since the majority of a laboratory control microprocessor's time is devoted to running experiments, collecting data, and calculating, the sharing of peripherals is not usually a problem. 
Digital Equipment Corporation (DEC) has prepared simple-to-use software that accommodates the hostsatellite organization described above. The software is called REMOTE-11, and it operates under the RT-11 operating system. In our application, a PDP-11/10 computer serves as the host. This computer is equipped with $24 \mathrm{~K}$ words of memory, 16 bits of digital $\mathrm{I} / \mathrm{O}$ buffering, A/D and D/A converters, a programmable real-time clock with Schmitt triggers, floppy disks, an RKO5 cartridge disk, and a line printer. The PDP-11/10 can serve as host to up to eight remote nodes. A node can be either a terminal or a satellite computer (usually accompanied by a terminal). The term "satellite" refers to a node that consists of a microprocessor and terminal. We currently have two satellites consisting of PDP-11/03 microprocessors for use in labs. Each is equipped with $20 \mathrm{~K}$ words of memory, 16-bit digital I/O buffers, and a terminal.

REMOTE-11 arbitrates the host computer's resources among the demands of as many as eight satellites and provides four services to the satellite user. First, an editor is provided that permits node users to edit files residing on the host's storage peripherals. Second, each satellite can request the host to load a program stored on the host's disk into the satellite. Third, REMOTE-11 provides a data-communications package for reliable data transmission between satellite and host. Finally, REMOTE-11 allows a node to use the host's background program partition. The advantages of this last feature will be described below.

These services are available if the REMOTE-11 is set up to run in the foreground of the host RT-11 system. The host's background is then available to an individual node user who wants access to the language processors (e.g., FORTRAN, MACRO, or BASIC compilers) or system peripherals. Access to the host's background is gained on a first-in demand basis.

The satellite microprocessors typically run in one of two modes, editing or program. Satellites can all run simultaneously in the editing mode, in the computing mode, or in any combination of these two modes. Editing or program preparation may be conducted with the REMOTE-11 text editor. When an editor user desires to compile his program or list it on the line printer, he requests use of the background portion of RT-11. If no one else is using the background, compiling may proceed or line-printer listing may occur as though the user had complete control of the host system.

The second mode of operation is the program mode. Satellite users store their programs on the host's disks. A satellite may request the host to load a program into the satellite. After loading, the satellite can disconnect from the host computer, thereby permitting completely independent computer control of an experiment. However, it is typically useful for the satellite to remain connected to the host so that data, collected by the satellite, may be shipped upstream to the host for pemanent disk storage. This would normally be done when available storage in satellite memory is exhausted.

The fact that DEC has a software package that permits the host-satellite configuration makes the LSI-1 1 and PDP-11/03 an attractive choice for moving into the world of microprocessors. Excluding the terminal, our microprocessor package cost about $\$ 2,000$ with $4 \mathrm{~K}$ of memory. (Other microprocessors can be obtained even more inexpensively, but we urge the reader to consider, honestly, engineering and software costs in pursuing al ternatives, especially if a host-satellite configuration is envisioned.)

To provide some idea of the REMOTE software commands, we list below the commands that are used by the satellite.

Initially, REMOTE-11 assumes that the user wants to be in the editing mode, so it types "FILE:". If the user wants to operate in the editing mode, he replies with the device name on which the file is stored, or will be stored, and then a file name (e.g., "DSK:PROG1.FOR" could prepare the file, PROG1.FOR, located on the RKO5 disk for editing.) Editing or creation of the file can then be undertaken.

If the editing mode is not desired, one of the six system commands can be entered. (1) Get: This command requests the host to find a program on a storage device and load it downstream into the satellite. (2) Start: This command starts a program in the satellite that was loaded with the "Get" command. (3) Run: This command implements both the "Get" and "Start" operations. (4) Send: This command sends a message to the terminal that currently occupies the background of RT-11. Accordingly, the current user of, for example, the FORTRAN compiler, can be notified that someone is waiting for the background. (5) Exit: This command puts the satellite user in the background mode of RT-11 so that compiling, printing, and so on, can occur. (6) R FLET: This command permits reentry of the satellite into a host's REMOTE-11 system from the background.

A disadvantage of any computer-sharing network is the absence of $100 \%$ guaranteed access to any shared device at any time. Only bottlenecks during actual experimental runs are usually at issue here (e.g., use of the line printer would not be time critical). The REMOTE-11 satellite-host system bypasses one typically important aspect of that problem. That is, for experimental computing and control purposes, each user does not share facilities, because he has his own computer. However, if a satellite were used to collect data and if memory capacity in the satellite could not accommodate all the data in an experimental run, then the user would need to pass data to the host for storage in a file, perhaps on a disk. Depending on the time requirement of the experiment, the experimenter might have to consider data-transmission rates.

We carried out a test on our microprocessor to determine how much time is required under REMOTE-11 
to request the host RKO5 disk to open a file and to store 200 four-digit numbers in that file from an array located in the microprocessor memory. One might, for example, collect 200 reaction times on 200 successive trials with the microprocessor and want to store these numbers in a disk file in order to free up satellite memory. When we transmitted these numbers from a FORTRAN program, in an A2 format, the time required was less than $1 \mathrm{sec}$. Obviously, the freeing up of memory space for this particular situation could be easily accomplished in an intertrial interval.

Complex laboratory functions can now be achieved quite inexpensively. Computer users should investigate the possibilities of gaining the advantages of individualized computing coupled with cost sharing of seldom-used and expensive peripherals.

(Accepted for publication June 19, 1978.) 